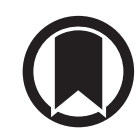

CrossMark

\title{
Transition from intravenous epoprostenol to selexipag in pulmonary arterial hypertension: a word of caution
}

\author{
To the Editor:
}

Prostacyclin analogues targeting the prostacyclin pathway are used to treat patients with pulmonary arterial hypertension (PAH), and are given either by an intravenous, subcutaneous or inhaled route [1]. Continuous i.v. infusion of epoprostenol has received the strongest recommendation for treatment of the most severe forms of PAH [2], mainly because it is the only PAH-specific drug that was demonstrated to give an improvement in survival in a randomised controlled study [3]. Due to drawbacks associated with its i.v. administration, epoprostenol is preferentially offered to young, high-risk patients [2]. For PAH patients in New York Heart Association (NYHA) functional class (FC) III/IV with severe haemodynamic impairment, epoprostenol can be combined with an endothelin receptor antagonist (ERA) and a phosphodiesterase type-5 (PDE-5) inhibitor to achieve sustained clinical and haemodynamic efficacy $[4,5]$.

Selexipag is a prostacyclin receptor agonist whose molecular structure is different from the prostacyclin analogues that can be orally administered with few side-effects $[6,7]$. Therefore, while selexipag has been developed for the treatment of $\mathrm{PAH}$, it is not considered equivalent to prostacyclin analogues $[1,2]$. Nevertheless, due to recurrent catheter-related infections and/or problems with patient acceptance, several physicians have attempted to replace i.v. epoprostenol with selexipag in selected patients. The current literature provides some reports and case-series of successful transitions from a prostacyclin analogue to selexipag. The available data are, however, fragmentary because of 1) the small number of cases; 2) the type of drug administered before the transition (mainly treprostinil (i.v. or s.c.) or inhaled prostacyclin); and 3) the absence of long-term haemodynamic follow-up. As not all prostacyclin analogues and/or all routes of administration are equivalent for the treatment of PAH [1], we aimed to investigate the consequences of transition from i.v. epoprostenol to oral selexipag.

The study population consisted of all patients with $\mathrm{PAH}$ enrolled in the French $\mathrm{PH}$ Network registry treated for at least 3 months with i.v. epoprostenol, who have been transitioned to oral selexipag, and with at least one evaluation, including a right heart catheterisation, while on selexipag. Clinical and haemodynamic characteristics of patients were collected at the time of first diagnosis of PAH, at the time of transition (while receiving epoprostenol, just before the initiation of selexipag), and at first and last evaluations on selexipag. Continuous variables were compared using a Wilcoxon signed-ranked test and categorical variables were compared using a McNemar test or a cumulative logic marginal model for variables with two categories and three or more categories, respectively [8]. A Bonferroni correction was applied to correct for multiple tests and, thus, a p-value lower than 0.025 was considered as significant.

We identified eight patients with PAH (idiopathic, $n=5$; heritable, $n=2$; associated with a connective tissue disease, $\mathrm{n}=1$ ) who were transitioned from i.v. epoprostenol to oral selexipag between September 2016 and January 2019 (six females; median age 39.4 (interquartile range (IQR) 34.1-52.7) years). All had a severe form of $\mathrm{PAH}$ at the time of diagnosis (figure 1).

At the time of transition, patients had been receiving i.v. epoprostenol for a median (IQR) 5.1 (2.0-8.3) years. The median (IQR) dose of epoprostenol was $17.5(15.0-29.5) \mathrm{ng} \cdot \mathrm{kg}^{-1} \cdot \mathrm{min}^{-1}$. All patients were also receiving an ERA and a PDE-5 inhibitor in combination with i.v. epoprostenol. They were all in stable condition and most were at "low risk" according to the French invasive and non-invasive approaches and

@ERSpublications

Even for patients who are very stable while receiving i.v. epoprostenol over a long period of time, any transition to selexipag warrants careful long-term clinical and haemodynamic follow-up because worsening is likely to occur http://bit.ly/32wR9eX

Cite this article as: Yanaka K, Guillien A, Soumagne T, et al. Transition from intravenous epoprostenol to selexipag in pulmonary arterial hypertension: a word of caution. Eur Respir J 2020; 55: 1902418 [https:// doi.org/10.1183/13993003.02418-2019]. 

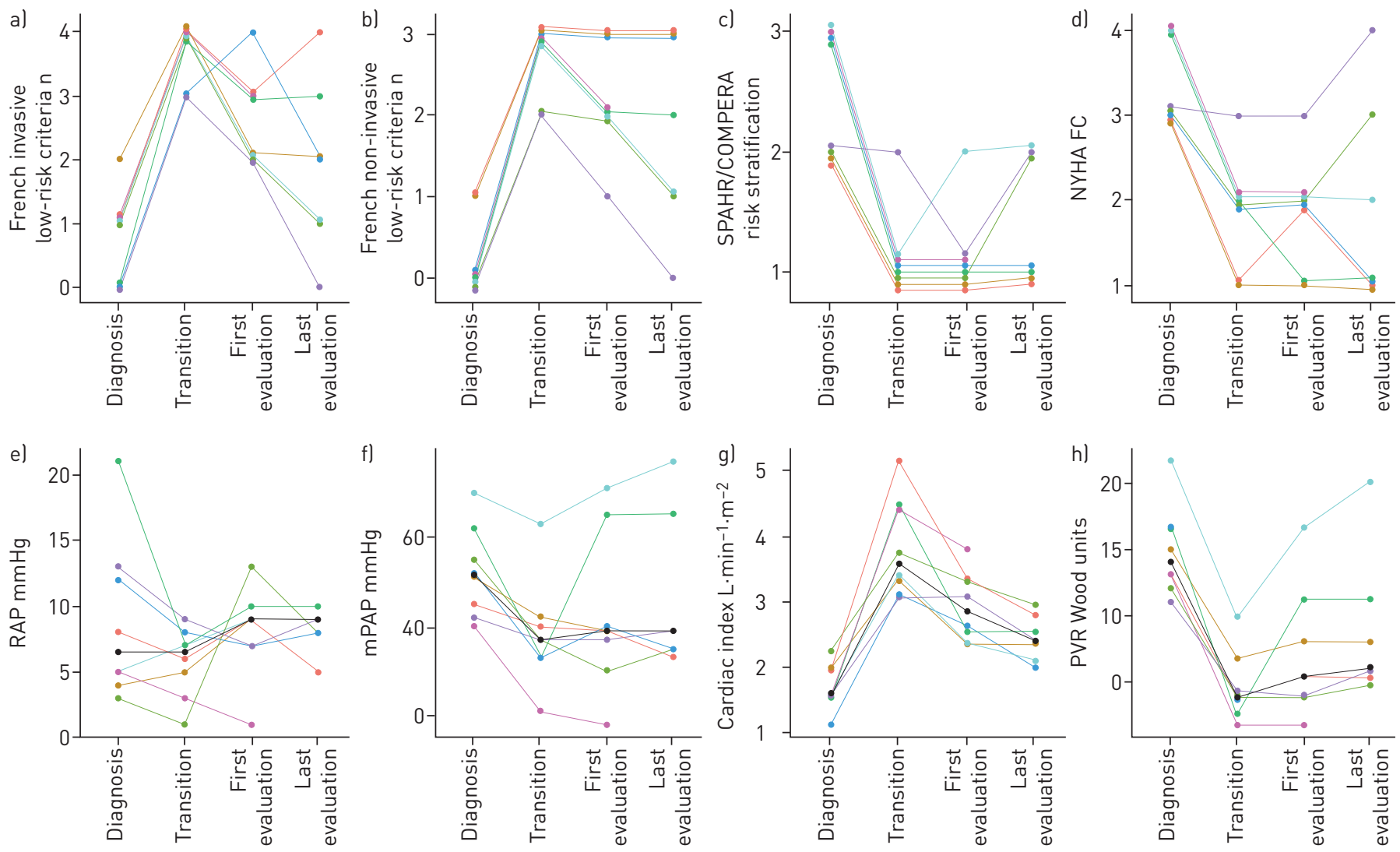

FIGURE 1 Individual clinical and haemodynamic characteristics of eight patients with severe pulmonary arterial hypertension (PAH) at diagnosis. Measurements at "diagnosis" were performed without any PAH treatment and measurements at "transition" in patients receiving a stable dose of intravenous epoprostenol. "First evaluations" were performed in patients receiving selexipag for a median (interquartile range) duration of 4.4 (4.0-6.3) months $(n=8)$ and "last evaluations" in patients receiving selexipag for 17.8 (6.1-25.9) months (n=7). a) Number of low-risk criteria met according to the French registry invasive method, the four criteria being 1) New York Heart Association (NYHA) functional class (FC); 2) 6-min walk distance (6MWD); 3) right atrial pressure (RAP); and 4) cardiac index. b) Number of low-risk criteria met according to French registry non-invasive method, i.e. 1) NYHA FC; 2) brain natriuretic peptide (BNP)/N-terminal pro-BNP (NT-proBNP); and 3) 6MWD. c) Risk stratification according to the SPAHR/COMPERA method, that grades 6MWD, NYHA FC, NT-proBNP, RAP, cardiac index and mixed venous oxygen saturation from 1 to 3 (1, low; 2, intermediate; 3, high) using the European Society of Cardiology/European Respiratory Society risk thresholds. d) NYHA FC. e-h) Individual values of RAP, mean pulmonary artery pressure (mPAP), cardiac index and pulmonary vascular resistance (PVR). The black lines indicate median values.

the SPAHR/COMPERA method (figure 1) [9-11]. Transition was decided either because of the patient's refusal to continue i.v. epoprostenol $(\mathrm{n}=2)$ or due to recurrent catheter-related infections $(\mathrm{n}=6)$. In all cases, selexipag dosage was uptitrated from $200 \mu \mathrm{g}$ b.i.d. to the maximal tolerated dose stepwise over 2 to 5 days, while parallel perfusion of epoprostenol was tapered off. The transition was well-tolerated in all cases. After transition and during follow-up, patients still received an ERA and a PDE-5 inhibitor together with a stable dose of selexipag ( $1600 \mu \mathrm{g}$ b.i.d. in all cases).

At the first evaluation on selexipag (4.4 (4.0-6.3) months after transition), NYHA FC and risk stratification (SPAHR/COMPERA method) did not change, but the risk assessment according to both French methods had significantly worsened compared with before transition ( $\mathrm{p}$-values of 0.024 and 0.023 for the invasive and non-invasive methods, respectively) (figure 1). In the meantime, 6-min walk distance (6MWD) and N-terminal prohormone of brain natriuretic peptide (NT-proBNP) remained remarkably stable (from $546(412-555)$ to $526(379-586) \mathrm{m}$ and from $190(136-354)$ to $224(152-598) \mathrm{ng} \cdot \mathrm{L}^{-1}$, respectively) but the cardiac index significantly decreased $(\mathrm{p}=0.016)$. The worsening of the French risk scores was mainly driven by a deterioration in cardiac index and in right atrial pressure (figure 1). At final evaluation on selexipag (17.8 (6.1-25.9) months after transition), 6MWD and NT-proBNP remained stable compared with the first evaluation (526 (304-619) $\mathrm{m}$ and $282(142-813) \mathrm{ng} \cdot \mathrm{L}^{-1}$, respectively). There was, however, a trend towards a further deterioration in risk and in cardiac index ( $\mathrm{p}$-values of $0.070,0.046$ and 0.049 for the French invasive, French non-invasive method and cardiac index, respectively) (figure 1). Between transition and last evaluation on selexipag, mixed venous oxygen saturation did not change 
significantly (from $73 \%(70-78 \%)$ to $68 \%(67-68 \%)$ ) despite the deterioration in cardiac index, and in the meantime serum haemoglobin increased by $2 \mathrm{~g} \cdot \mathrm{dL}^{-1}$.

To date, Frost et al. [12] have reported the largest series of patients $(n=34)$ undergoing transition from a prostacyclin analogue (treprostinil) administered by inhalation. Overall, patients in the series of FrosT et al. [12] remained stable throughout the 16-week treatment, but only clinical end-points were evaluated. In addition, as patients were long-term survivors (time from diagnosis close to 8 years, with a risk of a survivor bias), the follow-up of only 16 weeks was possibly too short to detect any decline.

Another series of four cases of transition from i.v. or s.c. treprostinil to selexipag has been reported. Transitions were considered successful but, unfortunately, only patients' conditions at transition were given, without any follow-up data [13]. These data are therefore of poor added value. Recently, HoLTHAUs et al. [14] reported a series of five patients with severe PAH at diagnosis. All were stable with a NYHA FC I/II while receiving i.v. treprostinil when they were transitioned to selexipag. Transition was well tolerated, with no significant short-term changes in NYHA FC. However, in line with our findings, there was a trend towards a reduction in cardiac index as early as 3-6 months after transition to selexipag, suggesting a poorer haemodynamic effect of selexipag than of i.v. treprostinil, but no long-term data were provided [14].

According to both our findings and the available literature, we suggest that some previous conclusions reporting stability after transition should be reconsidered, for at least two reasons. First, clinical criteria (such as 6MWD) may appear stable (as is the case in our series) while in the meantime, haemodynamic parameters (such as cardiac index) significantly worsen. Second, stability might be observed for transitions from less efficient forms of prostacyclin analogue treatments (i.e. inhaled administration) while significant deterioration is likely to occur for a transition from the most efficient treatment (i.e. i.v. epoprostenol). We therefore consider there is a need for a word of caution: even for patients who are very stable while receiving a given prostacyclin analogue (especially i.v. epoprostenol) over a long period of time, any transition to selexipag warrants careful long-term clinical and haemodynamic follow-up, because worsening is likely to occur. Such a transition should be restricted to patients unable to pursue epoprostenol for medical reasons, and only after a collegiate discussion. As far as possible, never change a winning team!

Kenichi Yanaka ${ }^{1}$, Alicia Guillien ${ }^{2}$, Thibaud Soumagne ${ }^{3}$, Justin Benet ${ }^{4}$, Nicolas Piliero ${ }^{1}$, François Picard ${ }^{5}$, Christophe Pison ${ }^{4}$, Olivier Sitbon $\oplus^{6,7,8}$, Hélène Bouvaist ${ }^{1}$ and Bruno Degano ${ }^{4,9}$

${ }^{1}$ Dept of Cardiology, Grenoble Alpes University Hospital, Grenoble, France. ${ }^{2}$ Environmental Epidemiology applied to Reproduction and Respiratory Health, INSERM, CNRS, University Grenoble Alpes, Institute for Advanced Biosciences (IAB), U1209 Joint Research Center, Grenoble, France. ${ }^{3}$ Dept of Intensive Care Medicine, University Hospital, Besançon, France. ${ }^{4}$ Dept of Respiratory Medicine, University Hospital of Grenoble Alpes, Grenoble, France. ${ }^{5}$ Dept of Cardiology, Haut-Lévêque, Pessac, France. ${ }^{6}$ University Paris-Saclay, Le Kremlin-Bicêtre, France. ${ }^{7}$ Dept of Respiratory Medicine, Bicêtre Hospital, AP-HP, Le Kremlin-Bicêtre, France. ${ }^{8}$ INSERM, UMR_S999, Le Plessis-Robinson, France. ${ }^{9}$ HP2 Laboratory, INSERM U1042, University Grenoble Alpes, Grenoble, France.

Correspondence: Bruno Degano, Service Hospitalier Universitaire Pneumologie Physiologie, CHU Grenoble Alpes, 38043 Grenoble Cedex, France. E-mail: bdegano@chu-grenoble.fr

Received: 16 Dec 2019 | Accepted after revision: 16 Feb 2020

Conflict of interest: K. Yanaka has nothing to disclose. A. Guillien has nothing to disclose. T. Soumagne has nothing to disclose. J. Benet has nothing to disclose. N. Piliero has nothing to disclose. F. Picard has nothing to disclose. C. Pison reports grants and personal fees from GlaxoSmithKline, personal fees from Novartis Pharma, Boehringer Ingelheim and AstraZeneca, outside the submitted work. O. Sitbon reports personal fees from Arena Pharmaceuticals, Acceleron Pharmaceuticals and Gossamer Bio, personal fees and non-financial support from Actelion Pharmaceuticals, grants and personal fees from Bayer HealthCare and Merck, non-financial support from GlaxoSmithKline, outside the submitted work. H. Bouvaist reports grants and non-financial support from GlaxoSmithKline and Bayer HealthCare, personal fees and non-financial support from Actelion Pharmaceuticals, outside the submitted work. B. Degano reports personal fees and non-financial support from Actelion Pharmaceuticals, non-financial support from Bayer HealthCare, grants, personal fees and non-financial support from Novartis Pharma, personal fees from Chiesi, GlaxoSmithKline and Menarini, outside the submitted work.

\section{References}

1 Barnes H, Yeoh HL, Fothergill T, et al. Prostacyclin for pulmonary arterial hypertension. Cochrane Database Syst Rev 2019; 5: CD012785.

2 Galie N, Channick RN, Frantz RP, et al. Risk stratification and medical therapy of pulmonary arterial hypertension. Eur Respir J 2019; 53: 1801889.

3 Barst RJ, Rubin LJ, Long WA, et al. A comparison of continuous intravenous epoprostenol (prostacyclin) with conventional therapy for primary pulmonary hypertension. N Engl J Med 1996; 334: 296-301.

4 D'Alto M, Badagliacca R, Argiento $P$, et al. Risk reduction and right heart reverse remodeling by upfront triple combination therapy in pulmonary arterial hypertension. Chest 2020; 157: 376-383. 
5 Sitbon O, Jais X, Savale L, et al. Upfront triple combination therapy in pulmonary arterial hypertension: a pilot study. Eur Respir J 2014; 43: 1691-1697.

6 Sitbon O, Channick R, Chin KM, et al. Selexipag for the treatment of pulmonary arterial hypertension. $N$ Engl J Med 2015; 373: 2522-2533.

7 Simonneau G, Torbicki A, Hoeper MM, et al. Selexipag: an oral, selective prostacyclin receptor agonist for the treatment of pulmonary arterial hypertension. Eur Respir J 2012; 40: 874-880.

8 Agresti A. An Introduction to Categorical Data Analysis. 3rd Edn. Hoboken, John Wiley and Sons, 2018.

9 Boucly A, Weatherald J, Savale L, et al. Risk assessment, prognosis and guideline implementation in pulmonary arterial hypertension. Eur Respir J 2017; 50: 1700889.

10 Hoeper MM, Kramer T, Pan Z, et al. Mortality in pulmonary arterial hypertension: prediction by the 2015 European pulmonary hypertension guidelines risk stratification model. Eur Respir J 2017; 50: 1700740.

11 Kylhammar D, Kjellstrom B, Hjalmarsson C, et al. A comprehensive risk stratification at early follow-up determines prognosis in pulmonary arterial hypertension. Eur Heart J 2018; 39: 4175-4181.

12 Frost A, Janmohamed M, Fritz JS, et al. Safety and tolerability of transition from inhaled treprostinil to oral selexipag in pulmonary arterial hypertension: Results from the TRANSIT-1 study. J Heart Lung Transplant 2019; 38: 43-50.

13 Fanous SM, Janmohamed M. Transition from treprostinil to selexipag in patients with pulmonary arterial hypertension: case series. Am J Health Syst Pharm 2018; 75: 1877-1881.

14 Holthaus N, Prins K, Rose L, et al. EXPRESS: transition from parental prostacyclin to selexipag: a case series of five pulmonary arterial hypertension patients. Pulm Circ 2019; 9: 2045894019862167.

Copyright @eES 2020 